\title{
Identification and Expression of Frizzled-3 Protein in Rat Frontal Cortex After Antidepressant and Electroconvulsive Treatment
}

\author{
Misa Yamada ${ }^{1,2}$, Tomoko Iwabuchi ${ }^{2}$, Kou Takahashi ${ }^{1,2}$, Chika Kurahashi $^{2}$, Hisayuki Ohata ${ }^{2}$, Kazuo Honda $^{2}$, \\ Teruhiko Higuchi ${ }^{3}$, and Mitsuhiko Yamada ${ }^{1}$ ** \\ ${ }^{1}$ Department of Psychogeriatrics, National Institute of Mental Health, National Center of Neurology and Psychiatry, \\ Tokyo 187-8502, Japan \\ ${ }^{2}$ Department of Pharmacology, School of Pharmaceutical Sciences, Showa University, Tokyo 142-8555, Japan \\ ${ }^{3}$ Musashi Hospital, National Center of Neurology and Psychiatry, Tokyo 187-8502, Japan
}

Received May 10, 2005; Accepted September 9, 2005

\begin{abstract}
The biological basis for the therapeutic mechanisms of depression are still unknown. While performing EST (expressed sequence tag) analysis to identify some molecular machinery responsible for the antidepressant effect, we determined the full-length nucleotide sequence of rat frizzled-3 protein (Frz3) cDNA. Interestingly, Northern blot analysis demonstrated that elevated levels of Frz3 were expressed continually from embryonic day 20.5 to postnatal 4 weeks in developing rat brain. In adult rat brain, Frz3 mRNA was expressed predominantly in the cerebral cortex and hypothalamus and moderately in the hippocampus. Using real-time quantitative PCR, we demonstrated that chronic treatment with two different classes of antidepressants, imipramine and sertraline, reduced Frz3 mRNA expression significantly in rat frontal cortex. Electroconvulsive treatment (ECT) also reduced Frz3 expression. In contrast, antidepressants and ECT failed to reduce Frz2 expression. Additionally, chronic treatment with the antipsychotic drug haloperidol did not affect Frz3 expression. Recently, the Frz/Wingless protein pathway has been proposed to direct a complex behavioral phenomenon. In conclusion, the Frz3-mediated signaling cascade may be a component of the molecular machinery targeted by therapeutics commonly used to treat depression.
\end{abstract}

Keywords: gene expression, mRNA fingerprinting, imipramine, sertraline

\section{Introduction}

Depression is one of the most prominent psychiatric diseases. Typical antidepressants acutely inhibit monoamine reuptake in nerve terminals, resulting in significant increases in the synaptic concentration of either noradrenaline or serotonin. However, a latency period of several weeks generally lapses before the clinical effects of antidepressants emerge. Repeated electroconvulsive treatment (ECT) is another therapy that is widely used, particularly in the treatment of drug-resistant depression. Although it has been known as an efficient treatment modality for decades, the basis of its therapeutic effects remains unknown. Its clinical effects also emerge over time.

*Corresponding author. FAX: +81-42-346-1994

E-mail: mitsu@ncnp-k.go.jp
The delayed clinical effects of antidepressants and ECT could result from either indirect regulation of neural signal transduction systems or regulation of gene transcription. Indeed, selective effects of antidepressants on specific immediate early genes and transcription factors have been described (1). These molecules may be important for adaptive neuronal changes resulting from chronic antidepressant treatment. Region-specific effects of chronic antidepressant treatment on the DNAbinding activities of CRE-, SP1-, and GRE-binding elements in rat hippocampus and frontal cortex are known (2). Taken together, these data demonstrate the possible role of gene expression alternations in the mechanism underlying antidepressant action. Therefore, quantifying these alterations occurring after chronic antidepressant treatment can help to identify novel molecular markers useful in the diagnosis and treatment 
of depression. Using differential cloning techniques, we and other groups have isolated genes that are differentially expressed in rat brain after chronic antidepressant treatment. To date, we have cloned several cDNA fragments as expressed sequence tags (ESTs), termed antidepressant-related genes, ADRGs $(3-6)$.

In the present study, we focused on ADRG\#78, an ADRG that encodes rat frizzled-3 protein (Frz3). The frizzled protein (Frz) family is a group of receptor proteins with seven putative transmembrane helixes (7). The N-terminal extracellular cysteine-rich domain of Frzs has been identified to be the ligand-binding domain that binds wingless proteins (Wnts), a family of secreted cysteine-rich glycosylated ligands (8). The Frz/Wnt pathway, first described in Drosophila, is a highly conserved developmental pathway involved in cell fate determination in the central nervous system of virtually all-eukaryotic organisms (9, 10). Recently, the Frz/Wnt pathway has been proposed to direct a complex behavioral phenomenon, and molecules participating in this pathway are encoded by candidate genes thought to be involved in neuropsychiatric disorders.

Here, we provide the first report identifying Frz3 in rat frontal cortex and describing its down-regulation after chronic treatment with antidepressants and ECT.

\section{Materials and Methods}

\section{Experimental animals}

Male Sprague Dawley rats (age: 7 - 10 weeks; Sankyo Labo Service Co., Tokyo) were housed in a temperaturecontrolled environment with a 12-h light/12-h dark cycle. They had free access to food and water. Rats were randomly separated into control and treated groups. Six rats were used for each treatment group. Experimental animals for antidepressant treatment received daily intraperitoneal injections of either vehicle, $10 \mathrm{mg} / \mathrm{kg}$ of imipramine (Sigma Chemical, St. Louis, MO, USA), or sertraline (Pfizer Pharmaceuticals, Inc., New York, NY, USA) dissolved in saline containing 1.5\% Tween-80; this injection protocol lasted for either 1 day or 21 days. As previously reported by our group, chronic treatment with $10 \mathrm{mg} / \mathrm{kg}$ of imipramine or sertraline reduced immobility in the water wheel test (3). Thus, it is proposed that both imipramine and sertraline administration in our protocol is adequate to induce an antidepressivelike effect in rats. Animals in the antipsychotic drug group were treated with haloperidol $(0.5 \mathrm{mg} / \mathrm{kg}$, Sigma Chemical) for 21 days. Animals in the ECT group were anesthetized with sevoflurane (Maruishi Pharmaceutical Co., Ltd., Osaka), and then they were given either a single electric shock (90 mA, 1.0-s duration) via ear-clip electrodes (single ECT group) or a series of electric shocks (90 mA, 1.0-s duration) every other day for 14 days (repeated ECT group). ECT was delivered with an Ugo Basile Model 7801 Unipolar, square-wave, electroconvulsive stimulation pulse generator (Stoelting Co., Wood Dale, IL, USA). Control rats were treated exactly as ECT-treated rats but received no electric current through the electrodes. Twenty-four hours after administration of the final antidepressant treatment or ECT, animals were euthanized by decapitation. The brain was quickly removed, dissected, and immediately frozen in liquid nitrogen and stored at $-80^{\circ} \mathrm{C}$ until use. All animal studies were carried out in accordance with protocols approved by the Showa University Ethics Committee in line with the OPRR Public Health Service Policy on Humane Care and Use of Laboratory Animals.

\section{EST analysis and $m R N A$ fingerprinting}

Total RNA from rat frontal cortex was extracted by Isogen reagent (Nippon Gene Co., Tokyo) according to the manufacturer's instructions. Isolated total RNA was dissolved in RNase-free water, and the concentration was estimated by UV spectrometry. Total RNA samples were treated with RNase-free DNase I (Nippon Gene Co.) for $30 \mathrm{~min}$ at $37^{\circ} \mathrm{C}$ and purified by phenol-chloroform extraction. The first strand cDNA was synthesized with reverse transcriptase (Invitrogen, Carlsbad, CA, USA), $1 \mu \mathrm{M}$ of oligo-dT primer, and $2 \mu \mathrm{g}$ of total RNA treated with DNase I and then diluted to a final volume of $100 \mu \mathrm{L}$. EST analysis was then carried out in the presence of $\left[{ }^{33} \mathrm{P}\right] \mathrm{dATP}$ (Life Science Products, Inc., Boston, MA, USA) with an mRNA fingerprinting kit (Clontech, Palo Alto, CA, USA), according to the manufacturer's instructions. Radiolabeled PCR products on denaturing $6 \%$ polyacrylamide gels were analyzed by electrophoresis. Three individual samples from each drug treatment group were applied side-by-side and visualized by autoradiography.

\section{Subcloning and sequence analysis}

The band of interest (ADRG\#78) was cut out of the dried gel, and the cDNA fragment was re-amplified using the same primer set used for mRNA fingerprinting (ATTAACCCTCACTAAATGCTGTATG, CATTATGCTGAGTGATATCTTTTTTTTTAC). The PCR conditions were as follows: denaturation at $94^{\circ} \mathrm{C}$ for $3 \mathrm{~min}$ followed by 40 cycles at $94^{\circ} \mathrm{C}$ for $30 \mathrm{~s}$, annealing at $60^{\circ} \mathrm{C}$ for $1 \mathrm{~min}$, and extension at $72^{\circ} \mathrm{C}$ for $1 \mathrm{~min}$. The re-amplified product was ligated into a pCR II-TOPO vector (Invitrogen) and transformed into competent TOP 10F' E. coli cells (Invitrogen). Sequence analysis was performed by standard dideoxy sequencing methods. Homology searches and sequence alignment were done using the FASTA search servers at the National Center 
Table 1. Oligonucleotides used for real-time quantitative PCR

\begin{tabular}{lll}
\hline mRNA & Forward primer & Reverse primer \\
\hline rat Frz3 & GACACAGCAGCATCCGAGACG & GGTTCATGCTGGTGCCAT \\
rat Frz2 & TGCACTCGTGGAGGAAGTTCT & CGCTTCACACGGTGGTCTCT \\
rat Frz1 & GCGCACCTGGATAGGCAT & TACTAGGTACGTGAGCACCGTGA \\
rat $\beta$-actin & TCGCTGACAGGATGCAGAAGG & GCCAGGATAGAGCCACCAAT \\
\hline
\end{tabular}

for Biotechnology Information. Additional cDNA sequences were determined by 3 '- and 5'-RACE-PCR using primer sequences derived from the partial cDNA sequences obtained from EST analysis.

\section{Northern blot analysis}

The cDNA fragment of rat Frz3 was amplified by PCR using a pair of $\left[{ }^{32} \mathrm{P}\right] \mathrm{dCTP}$-primers (5'-CGTCAC AAGATTCCGTAACCC-3' and 5'-CGGCATTATATC CCTAAAC-3') (Amersham Pharmacia Biotech, Tokyo). This amplified product was used as a probe for Northern blot analysis. Hybridization was carried out in ExpressHyb hybridization solution according to the manufacturer's instructions (Clontech). After hybridization, the membrane was exposed to X-ray film for $24 \mathrm{~h}$. To study the expression of Frz3, Rat Adult Tissue Blots (pre-made Northern blot nylon membrane from Seegene, Del Mar, CA, USA) were used for the Northern blot analysis. Total RNA isolated from tissues of SD rats, aged 8-12 weeks, was transferred onto the nylon membrane to make the "Rat Adult Tissue Blot". These tissues included brain, heart, lung, liver, spleen, kidney, stomach, small intestine, skeletal muscle, thymus, testis, nonimpregnated uterus, and placenta (20.5 days post-coitus). To study the developmental expression of rat Frz3 in the brain, Rat Brain Aging Blots (pre-made Northern blot nylon membrane from Seegene) were used. Total RNA from late embryonic and postnatal rat brain ( 20.5 days post-coitus and 1 day, 3 days, 1 week, 2 weeks, 4 weeks, 2 months, 3 months, 6 months, and 12 months postnatal) were isolated and transferred onto the nylon membrane to make the "Rat Brain Aging Blot". To study the expression pattern of Frz3 in the adult rat brain, Rat Brain Tissue Blots (premade Northern blot nylon membrane from Seegene) were used. Total RNA was isolated from olfactory bulb (including tubercle), cerebral cortex, hippocampus, thalamus, hypothalamus, midbrain, cerebellum, pons and medulla oblongata, and spinal cord were transferred onto the nylon membrane to make the "Rat Brain Tissue Blot".

\section{Real-time quantitative PCR}

Quantification of Frz expression in rat frontal cortex was performed with the real-time quantitative PCR method; this was carried out with an ABI PRISM 7000 instrument (Applied Biosystems, Foster City, CA, USA). PCR primers were designed by Primer Express Software (Applied Biosystems). A quantity of cDNA corresponding to $20 \mathrm{pg}$ of total RNA was amplified by PCR in duplicate. For each sample, three distinct amplifications were carried out in parallel. The primers used for rat Frz3, Frz2, Frz1, and $\beta$-actin, a reference for gene amplification, are indicated in Table 1. The SYBR $^{\circledR}$ Green PCR Core Reagents Kit (Applied Biosystems) was utilized for fluorescent detection of cDNA. For quantification, we used the Standard Curve Method (User Bulletin, ABI PRISM 7000 Sequence Detection System). Briefly, for rat Frzs and $\beta$-actin, an absolute standard curve was obtained by plotting the cycle of threshold $(\mathrm{Ct})$ following PCR amplification of serial dilutions of the control cDNA template. Data are presented as percentages of the control value (mean \pm S.E.M.). Differences were assessed using Student's $t$-test. A value of $P<0.05$ was regarded as statistically significant.

\section{Results}

\section{Identification and sequence analysis of rat $\mathrm{Frz} 3$}

We determined the nucleotide sequence of full-length ADRG\#78, one of the ADRGs we obtained from EST analysis. The original 395-bp cDNA fragment occupied positions $1518-1913$ in the full-length cDNA. Its open reading frame comprised 1998 nucleotides encoding a 666-residue polypeptide with a predicted molecular mass of $76.2 \mathrm{kDa}$. Homology analysis using the EMBL/GeneBank database revealed ADRG\#78 shares $95.2 \%$ and $88.5 \%$ homology with mouse and human Frz3 $(11,12)$, respectively, suggesting that ADRG\#78 is a rat homologue of mouse and human Frz3. The nucleotide sequence of full-length rat Frz3 cDNA can be found in the EMBL/GeneBank (accession number AF323956). Alignment of the deduced amino acid sequence of rat Frz3 with those of mouse Frz3, human Frz3, rat Frz1, and rat Frz2 is shown in Fig. 1. The deduced amino acid sequence of rat Frz3 was 99.4\% and $97.7 \%$ identical to those of mouse and human 


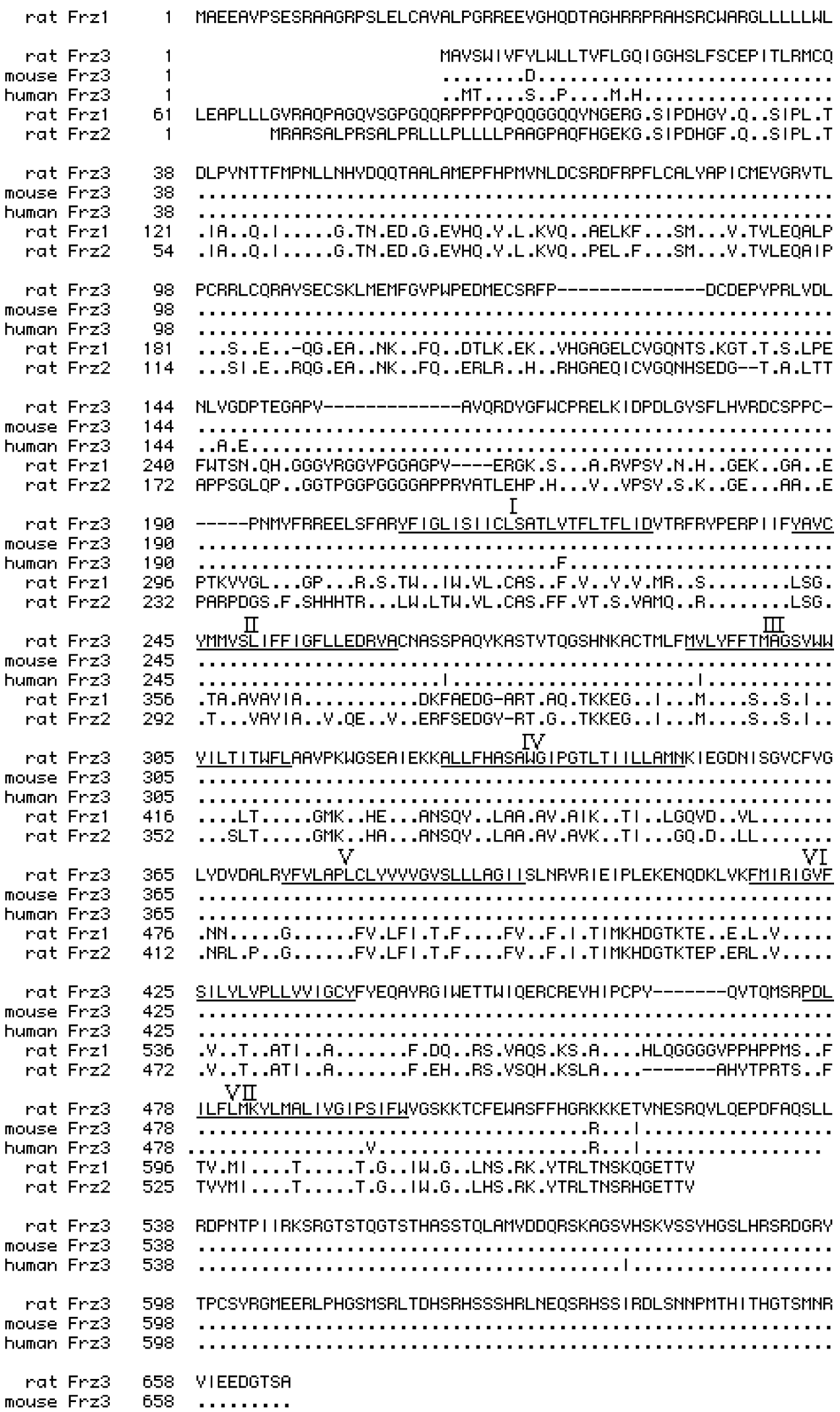

Fig. 1. Alignment of deduced amino acid sequences of rat Frz3, mouse Frz3, human Frz3, rat Frz1, and rat Frz2. Amino acid positions are numbered on the left. DDBJ/EMBL/GeneBank accession numbers are AF323956, U43205, AY005130, L02529, and L02530 for rat Frz3, mouse Frz3, human Frz3, rat Frz1, and rat Frz2, respectively. Dots represent identical amino acid residues found in rat Frz3. Predicted transmembrane domains are underlined. 


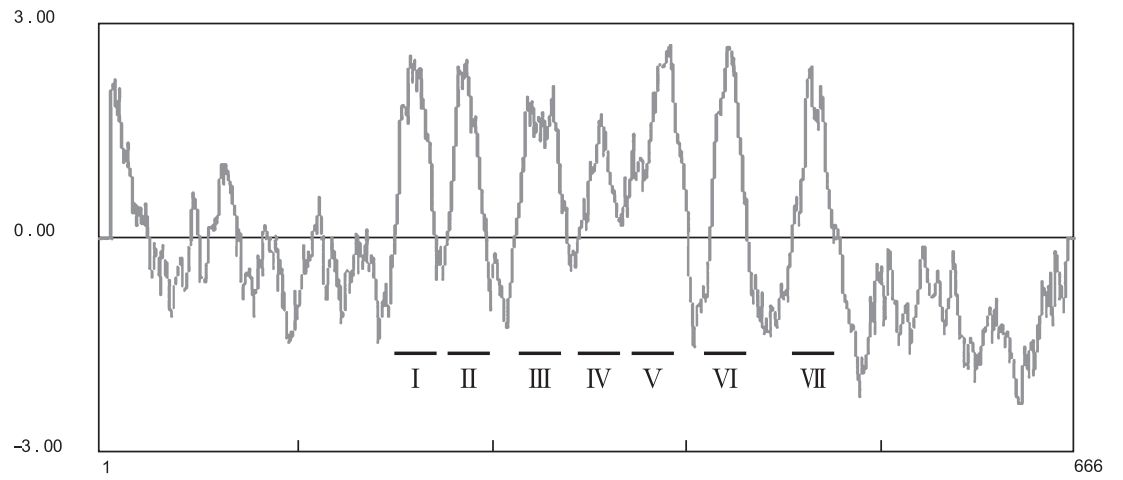

Fig. 2. Hydrophilicity/hydrophobicity analysis for rat Frz3 determined by the method of Kyte and Doolittle. Positive values denote regions of increased hydrophobicity. Putative transmembrane domains are indicated by solid lines. The presence of seven hydrophobic membrane-spanning stretches suggests Frz3 would be a member of the G-protein coupled receptor superfamily. These stretches are underlined in Fig. 1.

Frz3, respectively. On the other hand, the amino acid sequence homology of rat Frz3 to rat Frz1 and rat Frz2 (13) was only $50.6 \%$ and $44.5 \%$, respectively. As shown in Fig. 2, hydrophilicity/hydrophobicity analysis for rat Frz3 revealed the presence of seven hydrophobic membrane-spanning stretches, suggesting that it is a member of the G-protein coupled receptor superfamily. These stretches are underlined in Fig. 1.

\section{Expression of Frz3 $m R N A$ in the rat brain}

In adult rats, Frz3 mRNA was expressed widely in various tissues but to different degrees. Northern blot analysis with a $\left[{ }^{32} \mathrm{P}\right]-\mathrm{Frz} 3$ probe demonstrated that the $11.0-\mathrm{kb}$ transcript was in several tissues, including brain, kidney, and uterus. The $1.7-\mathrm{kb}$ transcript was primarily found in testis (Fig. 3A). The expression of Frz3 in the brain varied during development and aging. Expression levels from E20.5 to postnatal 4 weeks were relatively elevated. During postnatal development, Frz3 mRNA expression was present until at least 12 months of age and progressively diminished with age (Fig. 3B). In adult rats, Frz3 mRNA was expressed predominantly in the cerebral cortex and hypothalamus; moderately in the olfactory bulb and tubercle, hippocampus, thalamus, cerebellum, pons and medulla oblongata, and spinal cord; and weakly in the midbrain (Fig. 3C) . Expression of Frz2 mRNA in the rat brain was previously reported by another group (13).

Rat Frz3 mRNA expression determined by real-time quantitative $P C R$

We assessed the differential expression of Frz3 in antidepressant-treated and ECT-treated rats using realtime quantitative PCR and specific primers (Table 1). All expression data were normalized to the expression level of the housekeeping gene $\beta$-actin. As shown in Table 2, chronic treatment with either the tricyclic antidepressant imipramine or the selective serotonin reuptake inhibitor sertraline decreased Frz3 mRNA expression in the frontal cortex compared to control

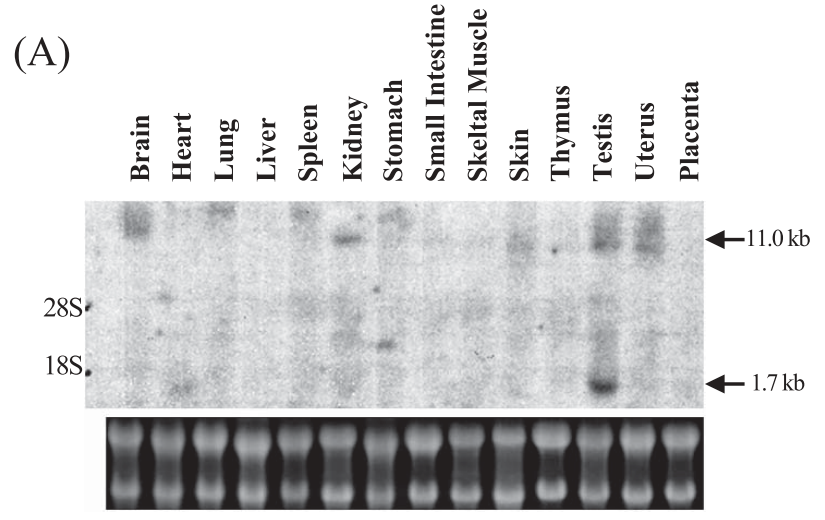

(B)
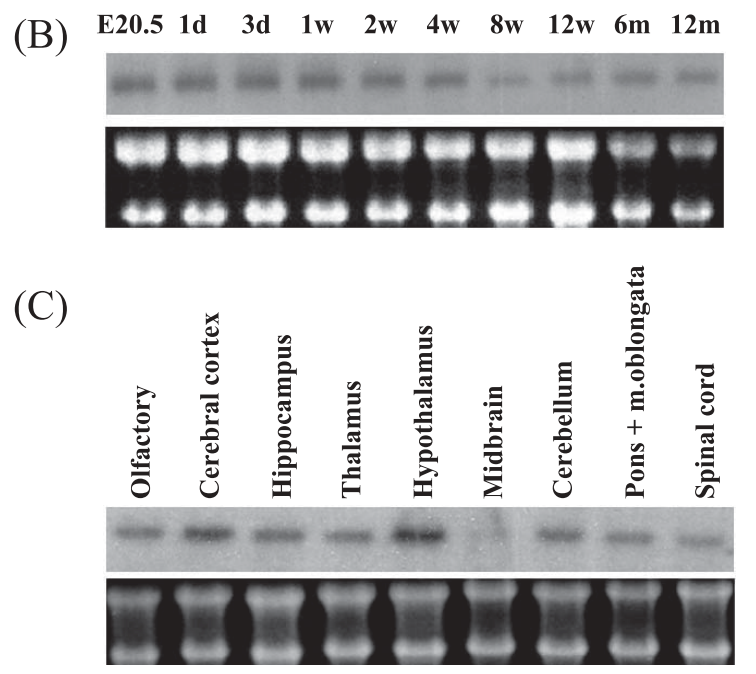

Fig. 3. Expression of Frz3 in rat tissues. Northern blot nylon membrane (Seegene) containing $20 \mu \mathrm{g}$ of total RNA extracted from various rat tissues (A), aged rat brain (B), and adult rat brain (C) were hybridized with $\left[{ }^{32} \mathrm{P}\right] \mathrm{dCTP}$-labeled Frz3-specific probe. Lower panels contain photographs of the ethidium bromide-stained gel, demonstrating similar levels of $18 \mathrm{~S}$ and $28 \mathrm{~S}$ ribosomal RNA as a loading control.

samples. Single-dose treatments with imipramine failed to affect Frz3 expression, whereas those with sertraline decreased expression (Table 2). This decrease, however, 
Table 2. Expression of rat Frz3 and Frz2 in the frontal cortex following antidepressant treatment, as determined by real-time quantitative PCR

\begin{tabular}{cllcc}
\hline & & Vehicle & Imipramine & Sertraline \\
\hline \multirow{2}{*}{ rat Frz3 } & acute & $100.0 \pm 9.0$ & $107.3 \pm 19.8$ & $72.2 \pm 10.0^{*}$ \\
& chronic & $100.0 \pm 17.1$ & $49.7 \pm 10.1^{*}$ & $62.4 \pm 16.1^{*}$ \\
rat Frz2 & acute & $100.0 \pm 16.5$ & $87.7 \pm 12.8$ & $91.3 \pm 15.5$ \\
& chronic & $100.0 \pm 10.9$ & $95.0 \pm 11.2$ & $86.8 \pm 9.3$ \\
\hline
\end{tabular}

Total RNA was extracted from frontal cortices of rats treated with either vehicle or $10 \mathrm{mg} / \mathrm{kg}$ of imipramine or sertraline for either 1 day or 21 days. Total RNA used for real-time quantitative PCR is described in the Materials and Methods. Data are percentages of control values (mean \pm S.E.M.). Differences were assessed by Student's $t$-test. A value of $* P<0.05$ was regarded as significant.

Table 3. Expression of rat Frz3 and Frz2 in the frontal cortex following ECT, as determined by real-time quantitative PCR

\begin{tabular}{cccc}
\hline & Control & Single ECT group & Repeated ECT group \\
\hline rat Frz3 & $100.0 \pm 10.8$ & $59.9 \pm 9.7^{*}$ & $54.5 \pm 7.2^{*}$ \\
rat Frz2 & $100.0 \pm 11.1$ & $138.1 \pm 10.3^{*}$ & $153.5 \pm 18.0^{*}$ \\
\hline
\end{tabular}

Total RNA was extracted from frontal cortices of sham rats or rats receiving either a single dose of ECT (90 mA, $1.0 \mathrm{~s})$ or repeated ECT $(90 \mathrm{~mA}, 1.0 \mathrm{~s}$, every other day for 14 days). Real-time quantitative PCR was performed as described in the Materials and Methods. Data are percentages of control values (mean \pm S.E.M.). Differences were assessed by Student's $t$-test. A value of $* P<0.05$ was regarded as significant.

was less than that observed in rats receiving chronic sertraline treatments. Interestingly, single-dose and repeat ECT significantly decreased Frz3 expression in the frontal cortex (Table 3). Although neither chronic nor single-dose administration of antidepressants affected Frz2 expression (Table 2), both single-dose and repeat ECT increased Frz2 expression (Table 3). We did not detect Frzl in either treatment condition (data not shown), perhaps because Frz1 expression in the frontal cortex is too weak to detect in adult rats (13). To determine the pharmacological specificity of these drugs, we examined the effect of a non-antidepressant antipsychotic drug, haloperidol, on Frz3 expression. Chronic haloperidol treatment failed to induce significant changes in Frz3 expression in the frontal cortex (data not shown), suggesting that the effects of imipramine and sertraline are specific.

\section{Discussion}

In the present study, we determined the full-length nucleotide sequence of rat Frz 3 cDNA. The pattern of rat Frz3 expression was similar to the patterns of mouse Frz3 and human Frz3 expression $(11,14)$. In adult rats, we found Frz3 mRNA in various tissues, but in different amounts. In the rat, Frz3 is transcribed into two major transcripts: an 11.0-kb mRNA in brain, kidney, and uterus and a 1.7-kb mRNA in testis (Fig. 3A). In humans, the Frz3 is also transcribed into two major transcripts, a $14.0-\mathrm{kb}$ mRNA in brain and a $1.8-\mathrm{kb}$ mRNA in pancreas (12). They concluded that the $1.8-\mathrm{kb}$ transcript encodes truncated Frz3 protein containing a $\mathrm{N}$-terminal cysteine-rich domain and four transmembrane domains, which permit the alternative polyadenylation of the mRNA at an internal AATAAA signal in the coding region. This internal signal also exists in rat Frz3 and is located in a similar region as that in rat Frz3 1321 CAAAGTGGGGCAGTGAAGCTATTGAGAAAAAAGCATTGCTGTTTCATGCCAGTGCCTGGG human Frz3 1430

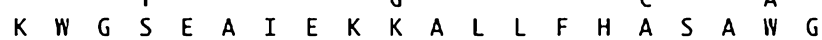

rat Frz3 1381 GCATCCCCGGAACTCTAACTATCATCCTITTAGCGATG ATAAA TTGAAGGTGACAATA human Frz3 $1490 * * * * * * * * * * * * * * * * * *(* * * * * * * * * * * * * * * * * * 1 * * * * * * * * * * * * * * * * * * * *$

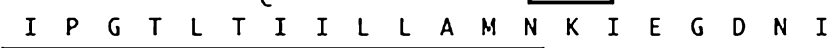

Fig. 4. Alignment of the nucleotide sequence for the fourth transmembrane domain and surrounding region of rat Frz3 and human Frz3. Nucleotide positions are numbered on the left. Asterisks represent identical nucleotides found in rat Frz3. The predicted fourth transmembrane domain is underlined. An alternative polyadenylation site is enclosed within a rectangle. 
human Frz3 (Fig. 4). Examination of rat Frz3 expression in brain during development and aging revealed that Frz3 is expressed continually at high levels from late embryogenesis (E20.5) to early postnatal development (postnatal 4 weeks) (Fig. 3B). This expression diminished with age. These findings suggest that Frz3 exerts profound effects on growth and development of the central nervous system.

In adult rat brain, Frz3 mRNA was expressed predominantly in the cerebral cortex and the hypothalamus and moderately in the hippocampus. Using real-time quantitative PCR, we demonstrated that Frz3 expression in rat frontal cortex decreased after chronic treatment with either imipramine or sertraline. Our data indicate that Frz3 would be one of the common functional molecules in the frontal cortex affected by the different types of chronic antidepressant treatments. Although it is possible that the therapeutic action on a single brain region underlies antidepressant treatment, it is also possible that pharmacological effects on multiple brain regions are responsible for the real therapeutic action of antidepressants and ECT. Studies to further characterize the neuronal circuitry of these brain regions will help elucidate the neuroanatomical substrates of antidepressive effects.

Interestingly, we also observed this altered pattern of Frz3 gene expression after both single-dose and repeated ECT. Single-dose treatments with imipramine failed to affect Frz3 expression, whereas those with sertraline decreased expression (Table 2). This decrease, however, was less than that observed in rats receiving chronic sertraline treatments. Together, the decreased Frz3 expression may initiate the adaptive neuronal changes resulting from chronic antidepressant treatment.

On the other hand, the non-antidepressant antipsychotic drug haloperidol, however, did not affect Frz3 expression. Moreover, Frz2 expression failed to decrease after chronic or single-dose administration of antidepressants, and rather, it increased after ECT. These results suggest that therapeutic treatments for depression specifically decrease Frz3 expression. The physiological role of the Frz2 induction after ECT is yet to be elucidated.

Interactions between Frz and certain Wnt ligands mediate specific downstream signals. Activation of the Frz/Wnt pathway leads to inactivation of glycogen synthase kinase (GSK-3 $\beta$ ) through the Dishevelledmediated pathway. GSK-3 $\beta$ normally promotes the turnover of $\beta$-catenin, a protein involved in synaptogenesis $(15,16)$, by direct phosphorylation, marking it for ubiquitylation and degradation in proteasomes. Therefore, the inhibition of GSK-3 $\beta$ leads to accumulation of $\beta$-catenin (10). Some researchers propose that $\beta$-catenin then enters the nucleus and binds LEF/TCF transcription factors; the $\beta$-catenin-LEF/TCF complex then acts as a transcriptional activator of Frz/Wnt target genes (17). In brain, $\beta$-catenin inactivation leads to structural malformation and craniofacial development failure (18). Dysfunction in the pathway also affects behavior. Transgenic mice overexpressing GSK-3 $\beta$ exhibit spatial learning deficits (19). It was recently proposed that the Frz/Wnt pathway participates in a complex behavioral phenomenon (20). Moreover, molecules involved in this pathway are encoded by candidate genes thought to be involved in certain neuropsychiatric disorders. Genetargeting of dishevelled-1, a cytoplasmic signaling protein that regulates $\beta$-catenin levels, shows that it influences social behavior and sensorimotor gating in mice (20).

Several pharmaceuticals commonly used to treat depression target components of this pathway. The direct inhibition of GSK-3 $\beta$ by lithium, an effective mood stabilizer for the treatment and prophylaxis of manic-depressive illness, leads to $\beta$-catenin stabilization both in vivo and in vitro $(21,22)$. Valproate, another mood stabilizer, regulates GSK-3 $\beta$-mediated axonal remodeling in developing neurons (22). GSK-3 $\beta$ is inhibited by phosphorylation on serine- 9 . It was recently demonstrated that serotonergic activity regulates the phosphorylation of GSK-3 $\beta$ in mouse brain. These results raise the possibility that impaired inhibitory control of GSK-3 $\beta$ may occur in conditions where serotonergic activity is dysregulated, such as in mood disorders (23). More recently, it is reported that a selective GSK-3 inhibitor AR-A014418 induces behavioral changes in rats, which are consistent with the effects of antidepressant medications (24). In addition, chronic ECT up-regulates $\beta$-catenin and induces $\beta$-catenin-associated cell division and differentiation in adult rat hippocampus (25). Detection of changes in the expression levels of molecules participating in the Frz/Wnt cascade may aid the diagnosis of depression or the ability to alter expression levels may contribute to the therapeutic efficacy of drugs used to treat depression.

In conclusion, we demonstrated here that Frz3 is commonly down-regulated following chronic antidepressant treatment or by ECT in rat frontal cortex. It is interesting to investigate the effect of non-monoaminergic candidates for antidepressants, including the non-peptidic selective delta-opioid receptor agonist SNC80 (26), on Frz3 expression. Also, further studies with a specific and sensitive antibody for rat Frz3 will be necessary to clarify the relationship between the Frz3mediated pathway and the therapeutic efficacy of antidepressant. 


\section{Acknowledgments}

Sertraline was kindly supplied by Pfizer Pharmaceuticals Inc., NY, USA. This work was in part supported by Health Science Research Grants from the Ministry of Health, Labor, and Welfare; the Japan Society for the Promotion of Science; Public Health Research Grant for Research on Serotonin; and the Mitsubishi Pharma Research Foundation.

\section{References}

1 Yamada M, Higuchi T. Functional genomics and depression research. Beyond the monoamine hypothesis. Eur Neuropsychopharmacol. 2002;12:235-244.

2 Frechilla D, Otano A, Del Rio J. Effect of chronic antidepressant treatment on transcription factor binding activity in rat hippocampus and frontal cortex. Prog Neuropsychopharmacol Biol Psychiatry. 1998;22:787-802.

3 Yamada M, Yamada M, Yamazaki S, Takahashi K, Nishioka G, Kudo K, et al. Identification of a novel gene with RING-H2 finger motif induced after chronic antidepressant treatment in rat brain. Biochem Biophys Res Commun. 2000;278:150-157.

4 Yamada M, Yamada M, Yamazaki S, Takahashi K, Nara K, Ozawa $\mathrm{H}$, et al. Induction of cysteine string protein after chronic antidepressant treatment in rat frontal cortex. Neurosci Lett. 2001;301:183-186.

5 Yamada M, Takahashi K, Tsunoda M, Nishioka G, Kudo K, Ohata $\mathrm{H}$, et al. Differential expression of VAMP2/synaptobrevin-2 after antidepressant and electroconvulsive treatment in rat frontal cortex. Pharmacogenomics J. 2002;2:377-382.

6 Takahashi K, Yamada M, Ohata H, Momose K, Higuchi T, Honda $\mathrm{K}$, et al. Expression of Ndrg2 in the rat frontal cortex after antidepressant and electro-convulsive treatment. Int $\mathrm{J}$ Neuropsychopharmacol. 2005;8:1-9.

7 Vinson CR, Conover S, Adler PN. A Drosophila tissue polarity locus encodes a protein containing seven potential transmembrane domains. Nature. 1989;338:263-264.

8 Dann CE, Hsieh JC, Rattner A, Sharma D, Nathans J, Leahy DJ. Insights into Wnt binding and signalling from the structures of two Frizzled cysteine-rich domains. Nature. 2001;412:86-90.

9 McMahon AP, Bradley A. The Wnt-1 proto-oncogene is required for development of a large region of the mouse brain. Cell. 1990;62:1073-1085.

10 Dale TC. Signal transduction by the Wnt family of ligands. Biochem J. 1998;329:209-223.

11 Wang Y, Macke JP, Abella BS, Andreasson K, Worley P, Gilbert DJ, et al. Large family of putative transmembrane receptors homologous to the product of the Drosophila tissue polarity gene frizzled. J Biol Chem. 1996;271:4468-4476.
12 Kirikoshi H, Koike J, Sagara N, Saitoh T, Tokuhara M, Tanaka $\mathrm{K}$, et al. Molecular cloning and genomic structure of human frizzled-3 at chromosome 8p21. Biochem Biophys Res Commun. 2000;271:8-14.

13 Chan SD, Karpf DB, Fowlkes ME, Hooks M, Bradley MS, Vuong V, et al. Two homologs of the Drosophila polarity gene frizzled (fz) are widely expressed in mammalian tissues. J Biol Chem. 1992;267:25202-25207.

14 Sala CF, Formenti E, Terstappen GC, Caricasole A. Identification, gene structure, and expression of human frizzled-3. Biochem Biophys Res Commun. 2000;273:27-34.

15 Nagafuchi A, Takeichi M, Tsukita S. The $102 \mathrm{kd}$ cadherinassociated protein: similarity to vinculin and posttranscriptional regulation of expression. Cell. 1991;65:849-857.

16 Nishimura W, Yao I, Iida J, Tanaka N, Hata Y. Interaction of synaptic scaffolding molecule and $\beta$-catenin. J Neurosci. 2002; 22:757-765.

17 Willert K, Nusse R. $\beta$-catenin: a key mediator of Wnt signaling. Curr Opin Genet Dev. 1998;8:95-102.

18 Brault V, Moore R, Kutsch S, Ishibashi M, Rowitch DH, McMahon AP, et al. Inactivation of the $\beta$-catenin gene by Wnt1Cre-mediated deletion results in dramatic brain malformation and failure of craniofacial development. Development. 2001; 128:1253-1264.

19 Hernandez F, Borrell J, Guaza C, Avila J, Lucas JJ. Spatial learning deficit in transgenic mice that conditionally overexpress GSK- $3 \beta$ eta in the brain but do not form tau filaments. J Neurochem. 2002;83:1529-1533.

20 Lijam N, Paylor R, McDonald MP, Crawley JN, Deng CX, Herrup $\mathrm{K}$, et al. Social interaction and sensorimotor gating abnormalities in mice lacking Dvl1. Cell. 1997;90:895-905.

21 Williams RS, Harwood AJ. Lithium therapy and signal transduction. Trends Pharmacol Sci. 2000;21:61-64.

22 Hall AC, Brennan A, Goold RG, Cleverley K, Lucas FR, Gordon-Weeks PR, et al. Valproate regulates GSK-3-mediated axonal remodeling and synapsin I clustering in developing neurons. Mol Cell Neurosci. 2002;20:257-270.

23 Li X, Zhu W, Roh MS, Friedman AB, Rosborough K, Jope RS. In vivo regulation of glycogen synthase kinase-3beta by serotonergic activity in mouse brain. Neuro-psychopharmacol. 2004;29: 1426-1431.

24 Gould TD, Einat H, Bhat R, Manji HK. AR-A014418, a selective GSK-3 inhibitor, produces antidepressant-like effects in the forced swim test. Int J Neuropsycho-pharmacol. 2004;26:1-4.

25 Madsen TM, Newton SS, Eaton ME, Russell DS, Duman RS. Chronic electroconvulsive seizure up-regulates $\beta$-catenin expression in rat hippocampus: role in adult neurogenesis. Biol Psychiatry. 2003;54:1006-1014.

26 Saitoh A, Kimura Y, Suzuki T, Kawai K, Nagase H, Kamei J. Potential anxiolytic and antidepressant-like activities of SNC80, a selective delta-opioid agonist, in behavioral models in rodents. J Pharmacol Sci. 2004;95:374-380. 